\title{
Recursos utilizados por Fisioterapeutas para Prevenção e Tratamento de Lesão por Pressão
}

\author{
Juliana Braga Facchinetti ${ }^{1}$; Fernanda Pires Fernandes ${ }^{2}$
}

Resumo: As Lesões por pressão são afecções graves, normalmente ocorrem em pacientes acamados, com algum déficit neurológico e/ou com alterações da sensibilidade. Essa lesão pode gerar várias complicações para pacientes hospitalizados e evoluir para uma infecção e até mesmo levar a morte. O estudo teve como objetivo avaliar os recursos utilizados por fisioterapeutas para prevenção e tratamento de úlceras por pressão em pacientes hospitalizados. Trata-se de um estudo descritivo com delineamento transversal e abordagem quantitativa. Foi aplicado um questionário estruturado aos Fisioterapeutas atuantes em determinados hospitais localizados no município de Vitória da Conquista - BA. Os dados foram tabulados e analisados através do software Excel 2015. Participaram da pesquisa 30 fisioterapeutas com idade média de 32 anos, tendo prevalência para sexo feminino, onde responderam um questionário sobre o conhecimento da prevenção e tratamento de lesão por pressão. Diante dos resultados encontrados pode-se considerar que os Fisioterapeutas atuantes em hospitais apresentaram carência de conhecimentos sobre a prevenção e tratamento de lesões por pressão.

PALAVRAS-CHAVE: Úlceras por pressão. Tratamento. Fisioterapia. Prevenção.

\section{Resources used by Physiotherapists for the Prevention and Treatment of Pressure Injury}

\begin{abstract}
Abastract: Pressure lesions are serious conditions, usually occuring in bedridden patients, with some neurological deficit and / or with alterations of the sensitivity. This condition can develop various complications for hospitalized patients and progress to an infection and even lead to death. The present study aims to evaluate the resources used by physiotherapists for the prevention and treatment of pressure ulcers in hospitalized patients. It is a descriptive study with a cross-sectional design and a quantitative approach. A structured questionnaire was applied to Physiotherapists working in certain hospitals located in the city of Vitória da Conquista - BA. Data was recorded and analyzed using Excel 2015 software. Thirty female physiotherapists with a average age of 32, with a prevalence for females, participated in the study, where they answered a questionnaire about the knowledge of prevention and treatment of pressure injury. In view of the results, it was found it can be considered that the Physiotherapists working in hospitals presented a lack of knowledge about the prevention and treatment of pressure injuries.
\end{abstract}

Keywords: Pressure ulcers. Treatment. Physiotherapy. Prevention.

\footnotetext{
${ }^{1}$ Graduação em Fisioterapia pela Universidade Católica de Salvador, especialização em Fisioterapia Dermato Funcional, atualmente é Docente da Faculdade Independente do Nordeste na cidade de Vitória da Conquista- BA. E-mail: julianafacchinetti@hotmail.com ${ }^{2}$ Graduação em Fisioterapia pela Faculdade Independente do Nordeste - FAINOR na cidade de Vitória da Conquista- BA. E-mail: fpnandesfisio@hotmail.com
} 


\section{Introdução}

A Lesão por Pressão (LP) é caracterizada como uma afecção em um determinado local da pele ou em qualquer área tissular, ela se desenvolve quando o tecido mole é comprimido, friccionado ou fica sobre pressão de alguma proeminência óssea por um longo período de tempo. Ela pode ser decorrente de fatores de risco que se diferenciam entre extrínsecos e intrínsecos, os fatores de risco extrínsecos são todos aqueles relacionados ao mecanismo de formação da LP, como a fricção, cisalhamento, umidade, imobilismo, higiene inadequada, colchão inadequado, e o uso da roupa de cama incorreto. Os fatores de risco intrínsecos são relacionados às variáveis do estado físico do paciente, como a mobilidade, habilidade diminuída, sensibilidade reduzida, idade, nutrição, hipotrofia, obesidade, uso de medicamentos depressores e edema (FREITAS et al., 2011).

As Lesões Por Pressões (LPs) são mais comumente afetadas na região sacral com incidência variável de $29,5 \%$ a 35,8\%, o calcâneo em segundo lugar com variação de 19,5\% e $27,8 \%$, logo depois ficando em terceiro lugar a região trocantérica com incidência variável entre $8,6 \%$ e 13,7\%. Em outras regiões do corpo como: glúteos, pernas, maléolos, pés, região isquiática, cotovelos, e escapulas, são menos acometidas tendo incidência variável entre $1 \%$ e 6\%. As LPs podem ocorrer também na região occipital, orelhas, mãos, região genital, apófises vertebrais, arcos costais, antebraço, abdômen, mama e nariz com incidência inferior a $1 \%$ (LUZ et al., 2010).

A prevenção da LP é a melhor solução para resolução do problema, sendo um importante assunto em todos os ambientes de cuidados de saúde. Esta afecção tem desafiado a sociedade por ser responsável em atrasar o retorno do paciente para casa, para suas atividades laborais, além de propiciar o desenvolvimento de sérias complicações. Para que a prevenção seja eficaz, primeiramente é de extrema importância avaliar os riscos com maior brevidade possível realizando, portanto, reavaliações. Deve ser feita uma abordagem estruturada que inclua uma avaliação da atividade, condições da pele, mobilidade, estado nutricional, nível de consciência, percepção sensorial, colher os dados sócio demográficos, dados clínicos, aplicar escalas que avaliem os riscos para desenvolvimento de LP, e assim programar medidas de prevenção especificas para cada paciente (LUZ et al., 2010). 
Os profissionais da área da saúde como médicos, enfermeiros e fisioterapeutas devem agir tanto de forma preventiva como curativa nas LPs. O fisioterapeuta deve realizar uma avaliação minuciosa do estado geral do paciente, observar a integridade da pele e a circulação sanguínea. Também é importante realizar mudanças de decúbito a cada duas horas, posicionamento adequado no leito, cinesioterapia, evitar contato direto com tecidos embolados ou muito grossos, evitar umidade, fricção e cisalhamento da pele, oferecer acolchoamento nas áreas de maior pressão para evitar danos teciduais, orientar quanto ao uso do colchão pneumático e a realizar crioterapia. Todas essas condutas são de grande importância para prevenção de lesões por pressão. (FURIERI et al., 2015).

No caso da lesão já instalada a fisioterapia tem como objetivo concentrar-se no processo de cicatrização da lesão podendo ser útil os recursos de eletroterapia, como a radiação infravermelha, ultrassom, laser de baixa intensidade, eletroestimulação de alta voltagem, corrente galvânica, além de todos os recursos de prevenção que vão auxiliar no aumento do aporte de nutrientes através do fluxo sanguíneo, facilitando o transporte de oxigênio aos tecidos, melhorando a reparação tecidual. Em casos mais avançados de LP, o indicado é realizar por profissionais específicos, o desbridamento, ostectomia e o fechamento da ferida, sendo importante também buscar o tratamento da patologia que gerou a LP (THULER; DANTAS, 2013).

As LPs podem ser responsáveis por retornos frequentes dos pacientes aos hospitais, por situações difíceis de sofrimento tanto para o portador quanto para os familiares, incluindo o grande percentual de morbidade e mortalidade notado na prática clínica. Essas situações causam alto custo para a família e/ ou governo, além de gerar mudanças no estilo de vida desses indivíduos (MIYAZAKI; CALIRI; SANTOS, 2010).

Destaca-se a necessidade da realização de estudos que abordem sobre os métodos de prevenção e tratamentos para LP em pacientes hospitalizados, com objetivo de gerar mais conhecimentos aos profissionais da área e menos custos para os familiares dos portadores e/ou governo, melhorando, por conseguinte, a qualidade de vida dos indivíduos. Dessa forma, o presente estudo teve como objetivo identificar os recursos utilizados por fisioterapeutas para prevenção e tratamento de lesão por pressão em pacientes hospitalizados. 


\section{Metodologia}

Trata-se de estudo descritivo com delineamento transversal e abordagem quantitativa. A pesquisa foi desenvolvida em três Hospitais localizados no município de Vitória da Conquista, que está situada na região sudoeste da Bahia, ocupando uma área territorial de $3204,257 \mathrm{Km}^{2}$, com população de 343.230 habitantes, sendo assim a terceira maior cidade do estado, e a quarta maior cidade do interior do Nordeste (IBGE, 2015). As instituições foram escolhidas por conveniência e facilidade de acesso as informações.

A população do presente estudo foi composta por Fisioterapeutas que estavam trabalhando nos Hospitais durante as visitas dos pesquisadores. Cada Hospital foi visitado dois dias diferentes da semana nos três turnos matutino, vespertino e noturno.

Foram incluídos no estudo todos os Fisioterapeutas que estavam trabalhando durante o período da coleta nos locais onde foi realizada a pesquisa portando a cédula de identidade profissional. Dentro dos critérios de exclusão estavam os Fisioterapeutas que não preencheram o Termo de Consentimento Livre Esclarecido (TCLE) e também profissionais que preencheram o questionário de forma incompleta.

Foi aplicado o Questionário de Informações Pessoais e Técnicas Utilizadas nos Pacientes Portadores de LP, este instrumento foi estruturado pelo pesquisador com objetivo de traçar o perfil dos Fisioterapeutas, contendo a identificação dos participantes, nome, idade, sexo, tempo de profissão e o tempo que trabalhavam na instituição. Além disso, o questionário também abordava sobre os recursos utilizados pelos Fisioterapeutas participantes da pesquisa para avaliação, prevenção e tratamento de lesão por pressão.

O questionário explorava questões sobre a frequência que os Fisioterapeutas realizavam avaliação da pele em seus pacientes, a frequência que realizavam mudanças de decúbito, se orientavam os familiares quanto à importância da utilização do colchão adequado, se possuíam informações sobre o estado nutricional do paciente, se protegiam as áreas acometidas pelas LP, entre outros aspectos.

A coleta de dados só deu início após a aprovação do Comitê de Ética e Pesquisa (CEP) da Faculdade Independente do Nordeste (FAINOR) com CAAE: 58936316.8.0000.5578. e Parecer $n^{\circ}$ 1.716.454. Foram obedecidos os princípios éticos da resolução 446/12 de acordo 
com o Conselho Nacional de Saúde (CNS). O abandono ou não aceitação em participar da pesquisa não provocou qualquer prejuízo aos Fisioterapeutas.

Inicialmente o participante recebeu esclarecimentos sobre o objetivo da pesquisa, como seriam realizados os procedimentos, os riscos e benefícios. Após ele compreender e concordar em participar de forma voluntaria, assinou o TCLE. Em seguida, o Fisioterapeuta foi dirigido a um local reservado e então foi aplicado o Questionário de Informações Pessoais e Técnicas Utilizadas nos Pacientes Portadores de LP, o pesquisador estava presente durante todo tempo, disponível para o esclarecimento sobre qualquer dúvida que pudesse surgir.

Os dados foram tabulados e analisados através do software Excel 2015. Foram verificadas as frequências e as medidas de tendência central (média, medianas, desvio padrão).

\section{Resultados}

Participaram da pesquisa 30 Fisioterapeutas que trabalhavam no Hospital São Vicente de Paulo 37\% ( $n=11)$, Hospital Geral de Vitória da Conquista 33\% ( $n=10)$ e IBR 30\% (n=9), a idade média dos participantes foi de 32 anos e o tempo de formação apresentou média de 7 anos. A maioria dos Fisioterapeutas era do sexo feminino $67 \%(n=20)$ e $43 \%(n=13)$ dos participantes eram especialistas na área de Unidade de Terapia Intensiva (UTI) (Tabela 1).

Tabela 01: Especialidades dos fisioterapeutas. Vitória da Conquista - BA, 2016.

\begin{tabular}{lcll}
\hline Caraterísticas & $\begin{array}{c}\text { \% } \\
\text { respostas }\end{array}$ & $\mathbf{N}$ & $\%$ \\
\hline Especialidade & 100 & & \\
UTI & & 13 & 43 \\
Ortopedia & 6 & 20 \\
Cardiorrespiratória & 3 & 10 \\
Saúde da família & 2 & 7 \\
Terapia manual & 2 & 7 \\
Hospitalar & 1 & 3 \\
Neurofuncional & 1 & 3 \\
Pilates & 2 & 7 \\
\hline
\end{tabular}

Fonte: Dados da pesquisa 
Na Tabela 2 encontram-se os resultados referentes às respostas do questionário sobre o conhecimento, a prevenção e abordagem terapêutica em LP. Todos participantes referiram ter conhecimento sobre lesão por pressão, e $97 \%(n=29)$ relataram ser importante avaliar a pele dos pacientes hospitalizados diariamente. Nos casos em que o paciente apresente hiperemia local, $63 \%$ (n=19) afirmaram ter o costume de realizar mudança de decúbito a cada duas horas e caso não ocorra o desaparecimento da hiperemia, 63\% (n=19) chamam a equipe de enfermagem para que ela decida o que fazer.

Quando questionados em relação a abordagem terapêutica deles em pacientes com lesões por pressão, todos responderam que realizavam como condutas a mudança de decúbito a cada duas horas, $90 \%(n=27)$ afirmaram posicionar o calcâneo do paciente com elevação e auxílio de coxim, 77\% (n=23) relataram ofertar orientações aos familiares quanto aos cuidados com os pacientes, 57\% $(\mathrm{n}=17)$ referiram amortecer o local de hiperemia, 53\% $(n=16)$ informaram realizar massagem no local e 37\% $(n=11)$ responderam fazer uso de luva d'água nas proeminências ósseas (Tabela 2).

Tabela 02: Conhecimento, prevenção e abordagem terapêutica em lesão por pressão. Vitória da Conquista, 2016. Vitória da Conquista - BA, 2016.

\begin{tabular}{lll}
\hline Variáveis & $\mathbf{N}$ & \% \\
\hline Conhecimento sobre lesão por Pressão & 30 & 100 \\
Sim & & \\
Não & 27 & 97 \\
Importância da avaliação da pele com qual frequência & 1 & 3 \\
Diariamente & & 63 \\
Duas vezes por semana & 19 & 23 \\
No caso de hiperemia local, você costuma & 7 & 3 \\
Realizar mudança de decúbito a cada duas horas & 1 & 10 \\
Realizar mudança de decúbito e reavaliar a pele após duas horas & 3 & 63 \\
Realizar mudança de decúbito e reavaliar a pele após meia hora & & 30 \\
Não realiza mudança de decúbito, responsabilidade da enfermagem & 19 & 7 \\
Se não houver o desaparecimento da hiperemia, você & 9 & \\
Chama a equipe de enfermagem para decidir o que fazer & 2 & 100 \\
Constata lesão tipo I e institui condutas & & 90 \\
Informa ao médico para receitar medicamento & 30 & 77 \\
Quais seriam as condutas & 27 & 57 \\
Mudança de decúbito a cada duas horas & 23 & 17 \\
Calcâneo elevado com auxílio de coxim & 16 & 53 \\
Orientação aos familiares sobre cuidados & 12 & 40 \\
Amortece local de hiperemia & 11 & 37 \\
Realiza massagem local & 11 & 37 \\
Minimiza exposição da pele a umidade & 7 & 23 \\
Evita o uso de fita adesiva sobre a pele & & \\
Usa luva com agua nas proeminências & & \\
Orienta utilizar sabonete de glicerina e hidratação da pele & & \\
\hline Fonte: Dados da pesquisa & & \\
\hline
\end{tabular}

Fonte: Dados da pesquisa. 
A maioria com 57\% $(n=17)$ dos participantes, afirmou não conhecer a Escala de Braden e $87 \%(n=26)$ afirmaram não fazer uso de qualquer escala para avaliar o risco de lesões por pressão. Em relação a orientar pacientes cadeirantes a realizarem alívio das áreas de pressão a cada 15 minutos, $67 \%(n=20)$ responderam que fazem esta orientação. Grande parte dos Fisioterapeutas $90 \%(\mathrm{n}=27)$ relatou utilizar algum método de prevenção para evitar o aparecimento de lesões por pressão (Tabela 3 ).

Quando questionados se a eletroterapia ajudava na cicatrização das lesões por pressão, $87 \%(n=26)$ dos Fisioterapeutas concordaram que seja eficaz na cicatrização da lesão por pressão, porém, o mesmo percentual não costuma utilizar este recurso terapêutico (Tabela 3).

Tabela 03: Conhecimento sobre a escala de Braden, a prevenção e abordagem terapêutica em lesão por pressão. Vitória da Conquista - BA, 2016.

\begin{tabular}{|c|c|c|}
\hline Variáveis & $\mathbf{N}$ & $\%$ \\
\hline \multicolumn{3}{|c|}{ Conhecimento da escala de Braden } \\
\hline Sim & 13 & 43 \\
\hline Não & 17 & 57 \\
\hline \multicolumn{3}{|c|}{ Utilização de alguma escala } \\
\hline Braden & 04 & 13 \\
\hline Nenhuma & 26 & 87 \\
\hline \multicolumn{3}{|c|}{$\begin{array}{l}\text { Orientar pacientes cadeirantes realizar alivio da pressão cada } 15 \\
\text { min }\end{array}$} \\
\hline Sim & 20 & 67 \\
\hline Não & 10 & 33 \\
\hline \multicolumn{3}{|c|}{$\begin{array}{l}\text { Você utiliza de métodos de prevenção para evitar o aparecimento } \\
\text { de Lp }\end{array}$} \\
\hline Sim & 27 & 90 \\
\hline Não & 03 & 10 \\
\hline \multicolumn{3}{|c|}{$\begin{array}{l}\text { É importante o planejamento multiprofissional baseado pelas } \\
\text { diretrizes }\end{array}$} \\
\hline Sim & 29 & 97 \\
\hline Não sei informar & 01 & 03 \\
\hline \multicolumn{3}{|c|}{ Conhecimento do colchão pneumático } \\
\hline Sim & 28 & 93 \\
\hline Não & 2 & 7 \\
\hline \multicolumn{3}{|c|}{ Concorda que eletroterapia ajuda na cicatrização } \\
\hline Sim & 26 & 87 \\
\hline Não & 04 & 13 \\
\hline
\end{tabular}


Id on Line Revista Multidisciplinar e de Psicoloqia

Id on Line Multidisciplinary and Psycology Journal

\section{Utiliza de algum recurso da eletroterapia}

Sim

Não

O hospital tem disponível algum aparelho de eletroterapia

Sim

Não

Qual recursos da eletroterapia você conhece a eficácia para lesão

Carboxiterapia

$02 \quad 07$

Laser de baixa intensidade

$24 \quad 80$

Tens

- $\quad-$

Infra vermelho

$04 \quad 13$

Ultra som

$12 \quad 40$

LED

Corrente galvânica

03

Fonte: Dados da Pesquisa

\section{Discussão}

As lesões por pressão estão diretamente associadas com altas taxas de morbidade e mortalidade principalmente em pacientes idosos e/ou por longo período acamados. Os principais fatores de risco incluem a imobilidade, as alterações cutâneas, a má nutrição e alteração cognitiva. Os Fisioterapeutas fazem parte da equipe multidisciplinar responsável pela prevenção do aparecimento de lesões por pressão. No presente estudo 30 Fisioterapeutas responderam o Questionário de Informações Pessoais e Técnicas Utilizadas nos Pacientes Portadores de LP, os resultados indicaram que todos participantes referiram ter conhecimento sobre lesão por pressão, e 97\% relataram ser importante avaliar a pele dos pacientes hospitalizados diariamente, corroborando com Rogenski e Kurcgant (2012), o qual afirma que a avalição da pele deve ser diária, para alerta de qualquer hiperemia local.

De acordo com Saraiva, Paula e Carvalho (2014) quando houver o aparecimento de hiperemia local, deve-se realizar mudança de decúbito e reavaliar a pele do paciente após meia hora, e se não houver o desaparecimento da hiperemia, deve-se constatar lesão por pressão grau I. Em contrapartida, apenas 3\%, ou seja, um participante, relatou realizar mudança de decúbito e reavaliar a pele após meia hora, 10\% afirmaram que não realizam 
mudança de decúbito por ser responsabilidade da enfermagem e $63 \%$ disseram realizar mudança de decúbito a cada duas horas.

Não ocorrendo o desaparecimento da hiperemia, 7\% dos participantes responderam informar ao médico para receitar algum medicamento, 63\% afirmaram que chamariam a equipe de enfermagem para decidir o que fazer e apenas $30 \%$ corroboram com Saraiva, Paula e Carvalho (2014) quando referiram constatar que o paciente estaria com lesão por pressão grau I e assim instituiriam as devidas condutas.

Todos os participantes quando questionados sobre as condutas necessárias nas lesões por pressão, responderam realizar mudança de decúbito a cada duas horas. Martins e Soares (2008) além de afirmarem que a mudança de decúbito evita a pressão prolongada e também a redução da irrigação sanguínea local, destacam ainda, a importância da hidratação da pele e do uso de sabonete de glicerina para prevenir o ressecamento e melhorar a elasticidade, evitando assim as fissuras na pele. No presente estudo, 23\% dos participantes relataram orientar o uso de sabonete de glicerina e hidratação após o banho.

Ao serem questionados sobre a utilização de fita adesiva em pacientes hospitalizados, apenas 37\% relataram evitar o uso da mesma sobre a pele. Quando se torna necessário o uso de fitas adesivas, é recomendado que se utilize um protetor de pele antes de colocar a fita adesiva pois a não utilização do protetor torna a pele mais frágil e susceptível a lesões (ROGENSKI; KURCGANT, 2012).

Lise e Silva (2007) alertam que o uso de luvas d'água é extremamente inadequado por causar umidade na pele podendo aparecer rachaduras, em contrapartida, $37 \%$ dos participantes demonstraram não ter este conhecimento ao afirmarem que utilizam luvas d'água nas proeminências ósseas dos pacientes. Ainda em relação a minimizar a exposição da pele do paciente a umidade, $40 \%$ dos fisioterapeutas relataram ter este cuidado.

De acordo com o Protocolo para Prevenção de Úlcera por Pressão (Brasil, 2013) a massagem é contraindicada na presença de inflamação aguda e onde existe a possibilidade de haver vasos sanguíneos danificados ou pele frágil. Dessa forma, a massagem não deverá ser recomendada como uma estratégia de prevenção de lesões por pressão, entretanto, no presente estudo 53\% dos participantes afirmou realizar a massagem no local.

A escala de Braden consiste em seis domínios: percepção sensorial, mobilidade, atividade, umidade, nutrição, fricção e cisalhamento; o escore pode variar de 6 a 23 pontos, é 
uma das escalas mais utilizadas na prevenção de lesão por pressão (SERPA et al., 2011). Todavia, 57\% dos participantes do estudo afirmaram não conhecer a escala de Braden, somente $13 \%$ confirmaram aplica-la em seus pacientes e $87 \%$ relataram não fazer uso de escalas para avaliação do risco de lesão por pressão.

No presente estudo, $90 \%$ dos participantes afirmaram utilizar de métodos preventivos nas lesões por pressão. Em relação aos pacientes cadeirantes $67 \%$ afirmaram orientar estes pacientes a realizarem alívio da pressão a cada 15 minutos. O Protocolo para Prevenção de Úlcera por Pressão (Brasil, 2013) afirma que a prevenção para o surgimento de lesões por pressão deve ser aplicada em qualquer paciente vulnerável, independente da faixa etária e as ações devem ser instituídas por todos os profissionais da área da saúde. A maioria dos casos dessas lesões pode ser evitada por meio da identificação dos pacientes em risco e da implantação de estratégias de prevenção confiáveis para todos os pacientes identificados como de risco. A grande maioria dos participantes (97\%) deste estudo confirmou a importância de se ter um planejamento pela equipe multiprofissional que se baseie em medidas adotadas pelas diretrizes de saúde.

Sobre conhecimento da eletroterapia, 87\% dos Fisioterapeutas concordaram que a eletroterapia ajuda na cicatrização das lesões por pressão. Mesmo assim, somente $13 \%$ relatou utilizar de recursos da eletroterapia para tratamento das lesões por pressão, sendo que $43 \%$ dos participantes tem o recurso de eletroterapia disponível em seu local de trabalho.

Sobre os recursos da eletroterapia que os participantes conheçam a eficácia no tratamento de lesão por pressão, $80 \%$ relaciona ao laser de baixa intensidade. Segundo Carvalho (2010), o laser de baixa intensidade apresenta efeitos sobre a formação de tecido de granulação, aperfeiçoa a vascularização e proliferação de fibroblastos, minimiza o efeito térmico gerando efeitos fotobiomoduladores que são importantes na cicatrização.

Em relação ao uso do ultrassom, $40 \%$ relataram ter o conhecimento sobre este recurso para tratar LP. Segundo Furieri et al. (2015), o ultrassom participa nas três fases do processo da lesão, estimula a proliferação fibroblástica na fase inflamatória, aumenta o aporte sanguíneo local, proporciona a formação de novos vasos sanguíneos, estimula o desempenho de fatores importantes para cicatrização, aumentando a elasticidade e fibras de colágeno.

Ainda sobre os recursos da eletroterapia em LP, 13\% dos Fisioterapeutas relacionaram a utilização do infravermelho. De acordo com Barbosa (2015), o infravermelho gera uma 
vasodilatação aumentando a circulação local, aumenta o metabolismo celular e auxilia na remoção de produtos indesejáveis na área acometida. O calor provoca a desidratação da pele proporcionando a reparação tecidual, porém, essa terapia deve ser realizada com muita cautela devido a diminuição da sensibilidade local, portanto é indicado o uso de um tecido úmido e uma dose baixa.

Apenas $7 \%$ dos participantes relacionaram a carboxiterapia para o tratamento das LP, entretanto, segundo Machado (2014), a carboxiterapia possibilita a melhora do fluxo sanguíneo, aumenta a oxigenação sanguínea cutânea, permite a nutrição celular, facilita a eliminação de produtos do metabolismo, favorece a produção de colágeno aumenta a espessura da derme contribuindo positivamente para uma boa cicatrização.

No que se refere ao uso da corrente galvânica para o tratamento de lesão por pressão, somente $10 \%$ dos participantes relataram ter conhecimento sobre o efeito deste recurso para LP. Segundo Furieri et al. (2015), a corrente galvânica é uma corrente do tipo polarizada onde possui efeitos eletroquímicos nas proximidades de seus polos, que produz efeitos alternados de polarização e despolarização tecidual tendo como efeito antiflamatório, analgesia, redução de edema, bacteriostático, eletroestimulação muscular, e através da fluidificação, hidratação e amolecimento da crosta da úlcera, favorece o processo de desbridamento e o processo de renovação tecidual.

Quando questionados sobre a utilização do TENS para LP, nenhum dos participantes relatou ter o conhecimento do seu efeito no processo cicatricial da lesão. Segundo Marques et al. (2016) o TENS acelera o processo de cicatrização através da inibição de citocinas pró inflamatórias, onde regulariza a formação de tecido de granulação, além disso promove a vasodilatação e estimulação neuromuscular, aumentando a perfusão dos tecidos, concentração de fibroblastos, redução do edema.

Não houve conhecimento dos participantes em relação ao uso de LED (Light Emiting Diodes), fototerapia comumente conhecido como ledterapia que traz como benefício a reparação e cicatrização tecidual. Segundo Chaves (2015) o LED consiste de ondas eletromagnéticas que gera a fotobiomodulação estimulando as funções celulares promovendo efeitos terapêuticos semelhantes ao efeito do laser, sendo importante no auxílio da cicatrização tecidual, pela facilidade de manuseio e possibilidade de ser organizado em 
matrizes de vários tamanhos. Ainda assim, 17\% dos participantes relataram não conhecer nenhum dos recursos da eletroterapia para tratamento de lesão por pressão.

A lesão por pressão pode gerar diversas consequências comprometendo a saúde e qualidade de vida do indivíduo. É importante que todos profissionais da saúde, inclusive os Fisioterapeutas, tenham conhecimentos necessários para prevenir e tratar as LP. O presente estudo apresentou limitações como a dificuldade de encontrar artigos científicos abordando diretamente a temática proposta, além da falta de interesse e/ou tempo dos Fisioterapeutas para responderem o questionário.

\section{Conclusões}

Os Fisioterapeutas participantes da pesquisa apresentaram uma média de sete anos de formação, a maioria era do sexo feminino e relatou ser importante realizar a avaliação da pele dos pacientes diariamente. Todos os Fisioterapeutas afirmaram ter conhecimento sobre LP, porém, a pesquisa identificou que a maior parte desses profissionais desconhecia a Escala de Braden, um instrumento importante que determina os riscos de desenvolvimento da LP. O maior número dos participantes referiu realizar massagem local como conduta para LP, sendo que este procedimento é contraindicado. $\mathrm{O}$ uso de luvas com água nas proeminências ósseas também faz parte erroneamente da prática de muitos desses profissionais. E apesar de grande parte dos participantes concordarem que a eletroterapia ajuda na cicatrização, apenas quatro Fisioterapeutas utilizam destes recursos em pacientes com LP.

Diante dos resultados encontrados, pode-se considerar que os Fisioterapeutas atuantes em hospitais apresentaram carência de conhecimentos sobre avaliação, prevenção e tratamento de LP, assunto este que deveria ser conhecido por todos profissionais de saúde e não somente pela enfermagem como citados por muitos deles.

Destaca-se a necessidade que a equipe de Fisioterapeutas atuante em hospitais conheça sua responsabilidade como profissionais de saúde, e se atualize adquirindo maiores conhecimentos das evidências atuais que constituem a avaliação, prevenção e tratamento da LP. Os resultados deste estudo puderam esclarecer e identificar as deficiências de informações 
dos Fisioterapeutas sobre o tema, sendo assim, novos estudos deverão ser realizados com intuito de criar estratégias para melhor informar e atualizar estes profissionais.

\section{Referências}

BATISTELA, M. A.; CHORILLI, M.; LEONARDI, G. R. Abordagens no estudo do envelhecimento cutâneo em diferentes etnias. Rev. Bras. Farm, v. 88, n. 2, p. 59-62, 2007.

CAMPOS, L. de L. R; ROSSI, V. E. C. Prevenção e tratamento de úlcera de pressão. Conexão ciência (Online), v. 5, n. 1, p. 92-104, 2010.

BARBOSA, F. K. A. A utilização dos recursos eletrotermofototerapêuticos no tratamento das úlceras diabéticas: uma revisão de literatura. 2015.

BAVARESCO, T; MEDEIROS, R. H.; LUCENA, A. de F. Implantação da Escala de Braden em uma unidade de terapia intensiva de um hospital universitário. Rev Gaúcha Enferm, v. 32, n. 4, p. 703-10, 2011.

BLANES, Leila et al. Avaliação clínica e epidemiológica das úlceras por pressão em pacientes internados no Hospital São Paulo. Rev Assoc Med Bras, v. 50, n. 2, p. 182-7, 2004.

DE CASTRO CARVALHO, Kelsyanne et al. Estudo da resistência cicatricial cutânea de ratos tratados com fototerapia a laser. ConScientiae Saúde, v. 9, n. 2, p. 179-186, 2010.

DE ABREU CHAVES, Maria Emília. Úlceras por pressão submetidas à fotobiomodulação com diodo emissor de luz. 2015.

DE FREITAS, Maria Célia et al. Úlcera por pressão em idosos institucionalizados: análise da prevalência e fatores de risco. Revista Gaúcha de Enfermagem, v. 32, n. 1, p. 143, 2011.

DE SOUZA, Diba Maria Sebba Tosta; DE GOUVEIA SANTOS, Vera Lúcia Conceição. Fatores de risco para o desenvolvimento de úlceras por pressão em idosos institucionalizados. Revista Latino-Americana de Enfermagem, v. 15, n. 5, p. 958-964, 2007.

FERNANDES, Natália Ciardulli Nunes; AMARAL, J. P. B. V. Conhecimento da equipe multidisciplinar sobre prevenção, avaliação e tratamento de úlcera de pressão no Hospital Universitário Sul Fluminense/RJ. Estação Científica-Edição Especial "Fisioterapia"[Internet], v. 1, n. 1, 2012.

FURIERI, Flávia Pignaton Morellato et al. ATUAÇÃO FISIOTERAPÊUTICA NA ÚLCERA POR PRESSÃO: UMA REVISÃO. Revista Científica FAEMA, v. 6, n. 1, p. 6980, 2015. 
GOMES, Flávia Sampaio Latini et al. Avaliação de risco para úlcera por pressão em pacientes críticos. Revista da Escola de Enfermagem da USP, v. 45, n. 2, p. 313-318, 2011.

GUIA RÁPIDO DA COLOPLAST, Um. Úlceras por pressão: Prevenção e tratamento, 2013.

LISE, Fernanda; DA SILVA, Lurdes Chiossi. Prevenção de úlcera por pressão: instrumentalizando a enfermagem e orientando o familiar cuidador e orientando o familiar cuidador. Acta Scientiarum. Health Sciences, v. 29, n. 2, p. 85-89, 2007.

LUZ, Sheila Rampazzo et al.. Geriatria \& Gerontologia, v. 4, n. 1, p. 36-43, 2010. Úlceras de pressão

MARQUES, Ana et al. Efetividade das correntes TENS (Transcutaneous Electrical Nerve Stimulation) na promoção do processo de cicatrização de feridas crónicas: revisão sistemática. 2016. Trabalho de Conclusão de Curso.

MARTINS, Dulce Aparecida; SOARES, Fabiana Fernandes Rego. Conhecimento sobre prevenção e tratamento de úlceras de pressão entre trabalhadores de enfermagem em um hospital de Minas Gerais. Cogitare Enfermagem, v. 13, n. 1, 2008.

MACHADO, Rafaela Martins. Emprego da Carboxiterapia no manejo do Fibro Edema Gelóide, Cicatrizes Atróficas e Flacidez de Pele. Journal of Applied Pharmaceutical Sciences-JAPHAC, v. 1, n. 2, p. 29-35, 2014.

MIYAZAKI, Margareth Yuri; CALIRI, Maria Helena Larcher; SANTOS, Claudia Benedita dos. Conhecimento dos profissionais de enfermagem sobre prevenção da úlcera por pressão. Rev Latinoam Enferm, v. 18, n. 6, p. 1203-11, 2010.

PINTO DA SILVA MORITA, Ana Beatriz et al. Ocorrência de Úlcera por Pressão em uma Instituição Hospitalar no Interior de São Paulo. Revista Eletrônica de Enfermagem do Vale do Paraíba, v. 1, n. 01, 2012.

RAMIRES, Rossinê Carvalho; MEJIA, Dayana Priscila Maia. Os efeitos do laser no tratamento de úlcera de decúbito-Revisão Bibliográfica. Fac Ávila-Ter Man, p. 1-12, 2012.

RESENDE, Daniella de Moura; BACHION, Maria Márcia; ARAÚJO, Lorena Aparecida de Oliveira. Integridade da pele prejudicada em idosos: estudo de ocorrência numa comunidade atendida pelo Programa Saúde da Família. Acta Paulista de Enfermagem, v. 19, n. 2, p. 168-173, 2006.

ROGENSKI, Noemi Marisa Brunet; KURCGANT, Paulina. Incidência de úlceras por pressão após a implementação de um protocolo de prevenção. Rev Lat Am Enfermagem, v. 20, n. 2 , p. $1-7$, 
SARAIVA, Isabella Leonetti; PAULA, Maria de Fátima Corrêa; CARVALHO, Rachel de. Úlcera por pressão no período transoperatório: ocorrência e fatores associados. Rev. SOBECC, v. 19, n. 4, p. 207-213, 2014.

SILVESTRE, Juliana Terezinha; HOLSBACH, Denise Rodrigues. Atuação fisioterapêutica na úlcera de pressão: Uma revisão de literatura. Revista Fafibe on-line. Bebedouro-SP, ano V, n. 5, p. 1-12, 2012.

WADA, Alexandre; NETO, Nuberto Teixeira; FERREIRA, Marcus Castro. Úlceras por pressão. Revista de Medicina, v. 89, n. 3/4, p. 170-177, 2010.

ZAMBONATO, Bruna Pochmann; ASSIS, Michelli Cristina Silva de; BEGHETTO, Mariur Gomes. Associação das sub-escalas de Braden com o risco do desenvolvimento de úlcera por pressão. Revista gaúcha de enfermagem. Porto Alegre. Vol. 34, n. 2 (jun. 2013), p. 2128.

Como citar este artigo (Formato ABNT):

FACCHINETTI, juliana B.; FERNANDES, Fernanda P. Recursos utilizados por Fisioterapeutas para Prevenção e Tratamento de Lesão por Pressão. Id on Line Revista Multidisciplinar e de Psicologia, 2017, vol.11, n.37, p. 421-435. ISSN: 1981-1179.

Recebido: 08.08.2017

Aceito: 12.08 .2017 